\title{
The cocktail infection: anaerobic and aerobic co-Infections in
}

\section{tubercular vertebral osteomyelitis [version 1; peer review: 1}

\section{approved with reservations, 1 not approved]}

\author{
Shyamasunder N Bhat (iD), Raghuraj Kundangar1, Nishanth Ampar (iD1, \\ Anika Sait(D1, Padmaja Ananth Shenoy1, Cynthia Amrutha Sukumar(D1, \\ Kavitha Saravu (iD) 1,2 \\ ${ }^{1}$ Kasturba Medical College, Manipal Academy of HIgher Education, Manipal, India
${ }^{2}$ Manipal Centre for Infectious Diseases (MAC ID), Prasanna School of Public Health, Manipal Academy of Higher Education, Manipal,
India
}

V1 First published: 01 Feb 2022, 11:130

https://doi.org/10.12688/f1000research.74393.1

Latest published: $09 \mathrm{Dec} 2022,11: 130$

https://doi.org/10.12688/f1000research.74393.2

\section{Abstract}

Background: Anaerobic organisms have been known to have an association with dental infections, bacteremia, endocarditis and soft tissue infections. However, anaerobic isolation from bone and joint infections are relatively rare. Sparse literature has been found on reports of anaerobic osteomyelitis. There is no literature reported on anaerobic osteomyelitis complicating Tuberculosis of spine.

Case Report: We report two cases of tuberculosis of spine complicated by aerobic and anerobic infections. The first is a case of a young female who presented with chronic lower backache and fever. Examination revealed a lumbar scoliosis with paraspinal tenderness. Magnetic resonance imaging (MRI) of the spine showed lumbar spondylodiscitis with multiple abscesses. There were air-fluid levels noted in the abscesses. The pus sent for CBNAAT (cartridge based nucleic acid amplification test) was positive. Further the cultures also grew Escherichia coli (aerobic) Bacteroids fragilis and Peptoniphilus asachrolyticus (anaerobic) organisms. She improved with a course of intravenous antibiotics and decompression surgery.

The second case is a middle aged man who presented with chronic neck pain and fever. Examination revealed kyphosis of the neck with spasm of the neck muscles and midline tenderness. MRI showed C4-5 cervical spondylodiscitis with parapharyngeal collections showing airfluid levels. The pus culture showed Streptococcus constellatus (aerobic) and Prevotella sps. (anaerobic). The CBNAAT report was positive for Mycobacterium tuberculosis. The patient was treated with intravenous antibiotics and cervical decompression.

Conclusion: Though tubercular vertebral osteomyelitis (TVO) is usually a diagnosis in itself, it should not hinder us from considering
Open Peer Review

Approval Status $\times ?$

1 2

version 2

(revision)

09 Dec 2022

version 1

01 Feb 2022

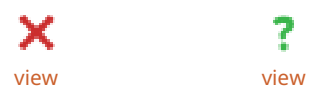

1. Kaouther Maatallah, University Tunis el Manar, Manouba, Tunisia

2. Gazanfar Rahmathulla (D), University of Florida Health, University of Florida Health Jacksonville, USA

Any reports and responses or comments on the article can be found at the end of the article. 
secondary infections (both aerobic and anaerobic) complicating the osteomyelitis. Further, the presence of air-fluid levels on imaging studies and the presence of foul smell during operative exploration of the spine must arouse the suspicion of an anaerobic co-infection. Isolation and treatment of these organisms are crucial as they may hamper the clinical outcome of the primary TVO.

\section{Keywords}

Anaerobic osteomyelitis, Prevotella, Bacteroides, Peptostreptococcus, spondylodiscitis, tuberculosis of Spine, Tubercular Vertebral

Osteomyelitis

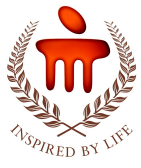

This article is included in the Manipal Academy

of Higher Education gateway.

\section{Corresponding author: Cynthia Amrutha Sukumar (cynthiaamrutha@gmail.com)}

Author roles: Bhat SN: Conceptualization, Data Curation, Investigation, Methodology, Project Administration, Resources, Supervision, Validation, Writing - Original Draft Preparation, Writing - Review \& Editing; Kundangar R: Conceptualization, Data Curation, Resources, Supervision, Writing - Review \& Editing; Ampar N: Data Curation, Formal Analysis, Methodology, Writing - Review \& Editing; Sait A: Data Curation, Resources, Visualization, Writing - Original Draft Preparation; Shenoy PA: Data Curation, Formal Analysis, Resources,

Supervision, Writing - Original Draft Preparation; Sukumar CA: Conceptualization, Data Curation, Investigation, Methodology, Writing Original Draft Preparation, Writing - Review \& Editing; Saravu K: Conceptualization, Methodology, Resources, Supervision, Writing Review \& Editing

Competing interests: No competing interests were disclosed.

Grant information: The author(s) declared that no grants were involved in supporting this work.

Copyright: @ 2022 Bhat SN et al. This is an open access article distributed under the terms of the Creative Commons Attribution License, which permits unrestricted use, distribution, and reproduction in any medium, provided the original work is properly cited.

How to cite this article: Bhat SN, Kundangar R, Ampar $\mathrm{N}$ et al. The cocktail infection: anaerobic and aerobic co-Infections in tubercular vertebral osteomyelitis [version 1; peer review: 1 approved with reservations, 1 not approved] F1000Research 2022, 11 :130 https://doi.org/10.12688/f1000research.74393.1

First published: 01 Feb 2022, 11:130 https://doi.org/10.12688/f1000research.74393.1 


\section{Introduction}

Anaerobic organisms have been known to have an association with dental infections, bacteremia, endocarditis and soft tissue infections. ${ }^{1}$ However, anaerobic isolation from bone and joint infections are relatively rare. This could be attributed to the fastidious nature of the organism which makes isolation from the bone and joint infections cumbersome. ${ }^{1}$

The most common organisms implicated in anaerobic osteomyelitis are Bacteroides, Fusobacterium, Peptostreptococcus and Clostridium species. Predisposing factors include children, contiguous spread of infection, vascular disease and complicated fractures. ${ }^{2}$ Sparse literature has been found on reports of anaerobic osteomyelitis. There is no literature reported on anaerobic osteomyelitis complicating Tuberculosis of spine/Tubercular vertebral osteomyelitis (Pott's spine). However, our cases highlight the rare isolation of gram-negative anaerobes (Prevotella, Bacteroides, Peptoniphilus) from Tubercular vertebral osteomyelitis (TVO) in immunocompetent adults diagnosed with tuberculosis of spine.

\section{Case report}

Case 1

A 22-year-old poorly built (body mass index: $16 \mathrm{~kg} / \mathrm{m}^{2}$ ) lady from rural South India who was pursuing her undergraduate degree, presented with complaints of low back ache of one-month duration. It was insidious in onset and gradually radiated to the right gluteal region. She was able to walk only a few steps with a limp. There was no bowel or bladder disturbance. She preferred to keep her right hip flexed to about 30 degrees in supine position. The backache was associated with high grade fever with chills and rigors. There was no history of tuberculosis in her family or her community. She had no significant history suggestive of immunosuppression. She had no known co-morbidities. There was no significant genetic history given by the patient.

On examination, she was febrile $\left(102^{\circ} \mathrm{F}\right)$ with a pulse rate of 98 beats/minute. There was midline and right paraspinal tenderness from L3 to sacral region. Neurological examination of lower limbs was unremarkable.

Lab investigations showed that she was anaemic (Haemoglobin of $8 \mathrm{~g} / \mathrm{dl}$ ). Her total counts were 13600/mm ${ }^{3}$ (predominantly neutrophilia). The ESR was elevated $(88 \mathrm{~mm} / \mathrm{hour})$ and CRP $(261 \mathrm{mg} / \mathrm{L})$. The renal function tests were within normal limits, while serum albumin was only $1.9 \mathrm{mg} / \mathrm{dl}$. Blood and urine cultures were sterile.

A radiograph revealed mild lumbar scoliosis to the left side and bulky psoas muscle in the right side (Figure 1a and $\mathrm{b}$ ).

MRI spine revealed features suggestive of spondylodiscitis of L5-S1, spondylitis of L4 with abscesses adjacent to L4, L5, $\mathrm{S} 1, \mathrm{~S} 2$ and S3 vertebra in the right side. There was also air-fluid level in the abscess (Figure 1c and d).

She underwent decompression of L5 vertebra and exploration of L5-S1 space with drainage of the abscess. Intraoperatively foul-smelling gas escaped from the abscess. The L5-S1 disc also had purulent collection. Pus was sent for CBNAAT (cartridge based nucleic acid amplification test) for tuberculosis, aerobic and anaerobic cultures. Material from L5-S1 disc was sent for histopathology.

On the third hospital day CBNAAT report was suggestive of Mycobacterium tuberculosis she was started on anti-tubercular therapy (ATT). The next day the aerobic culture grew Escherichia coli whereas anaerobic culture grew Bacteroids fragilis and Peptoniphilus asachrolyticus. In addition, she was also started on injection PiperazillinTazobactem and oral tinidazole for 10 days. There was wound dehiscence on tenth postoperative day.

The repeat culture from the pus drained grew only $E$. coli with similar sensitivity though the growth was scanty. We continued antitubercular drugs and added Injection Amikacin and Imipenem (as per the culture sensitivity report) for a total of three weeks. She was taken up for secondary suturing during this period. Patient was discharged subsequently. On follow-up at one month, her symptoms had improved and her anemia had improved to $9.5 \mathrm{gm} \%$. She had also gained $1.6 \mathrm{kgs}$ in weight. Repeat radiographs and MRI were done at the three-month follow-up and showed resolution of the abscesses. Patient was adherent and tolerated the anti-tubercular medications well.

Case 2

A 54-year-old man from an urban district of South India, a tailor by occupation, came with complaints of insidious onset of neck pain of about three weeks duration. The pain was gradually progressive over the past 10 days. The pain would aggravate on movements of the neck, but there was no radiculopathy. There was also associated fever with chills and rigors. He reported significant weight loss in the recent past. There was no history of medical co-morbidites. There was no relevant psycho-social or genetic history obtained from the patient. 

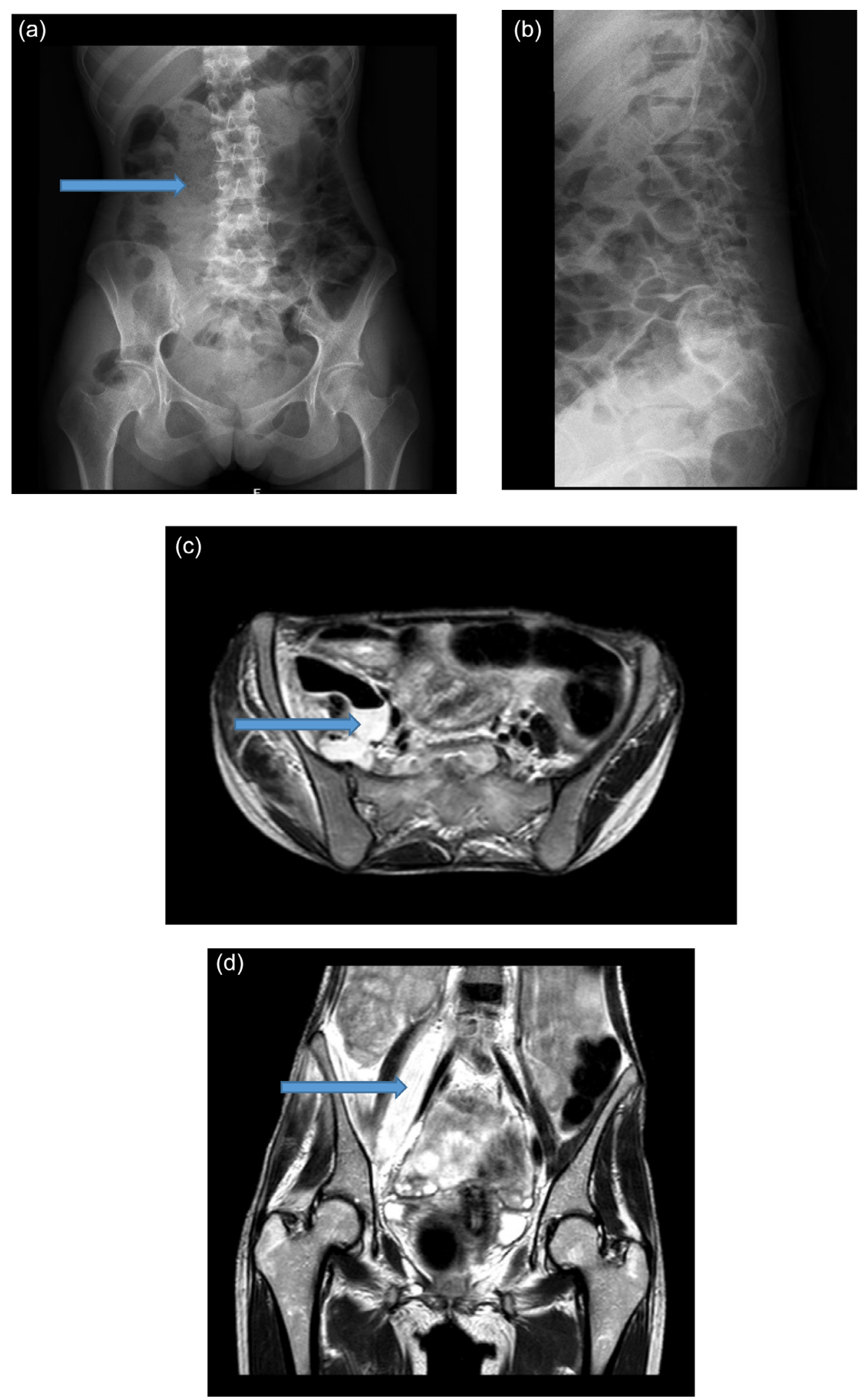

Figure 1. a: Radiograph of Spine (AP view). Arrow indicating bulky psoas muscle. b: Radiograph of Spine (lateral view). c: MRI (T2 flair) transverse section lumbar spine. Arrow indicating Abscess with air-fluid level. d: MRI lumbar spine. Saggital section. Arrow indicating right psoas abscess. 
On examination, neck appeared short and kyphotic. There was midline tenderness from C4-C7 region. Movements of the neck was grossly restricted due to pain and spasm. The elbow flexion was MRC Grade $4 / 5$ on both sides. There was no sensory deficits and reflexes were normal. He was anaemic $(\mathrm{Hb} 10 \mathrm{~g} / \mathrm{dl})$ with neutrophilia. ESR was moderately elevated at $68 \mathrm{~mm} / \mathrm{hour}$.

In an X-ray of the cervical spine, we noted increased prevertebral soft tissue shadow and erosion of vertebral end plates at C4-5 level with gross kyphosis. Air-fluid levels were also seen in the prevertebral soft tissue shadow (Figure 2a and b).

MRI of the cervical spine confirmed spondylodiscitis of C4-C5 with cord compression. Multiple collections in parapharyngeal space communicating with the pre-vertebral space were also seen along with air-fluid levels (Figure $2 \mathrm{c}$ and d)

By this time the blood culture grew Streptococcus constellatus and he was started on Injection ceftriaxone (2gm q24h) as per sensitivity report. His urine culture was sterile and Brucella agglutination test was negative.

He underwent anterior cervical decompression, by C5 corpectomy and fusion (Figure 2e and f). A distinct foul-smelling odour was noted intra-operatively and around $100 \mathrm{ml}$ of pus was drained. The C4 and C5 vertebrae were completely destroyed with surrounding caseous material. Pus was sent for aerobic and anaerobic cultures and the caseating tissue was sent for histopathology. Patient tolerated the procedure well.

The aerobic bacterial culture from the pus grew Streptococcus constellatus with similar sensitivity as that of his blood culture. Anaerobic culture grew Prevotella sps. The CBNAAT report was positive for Mycobacterium tuberculosis. Histopathology confirmed granulation tissue with epitheloid cells and Langhans giant cells suggestive of tubercular osteomyelitis.

He was started on Antitubercular therapy and Injection Metronidazole (500 mg q8h for two weeks) in addition to Injection Ceftriaxone (two weeks). The wound healed well. Antitubercular therapy is being continued and patient is doing well at two months follow up. Improvement in anemia and ESR as well as weight gain was noted in this patient.

\section{Consent}

Written informed consent for publication of their clinical details and/or clinical images was obtained from the patients.

\section{Discussion}

Anaerobic osteomyelitis was first reported in 1884 by Von Langenbeck in case of vertebral osteomyelitis. ${ }^{3}$ Decades later, Taylor and Davies noted the presence of anaerobic organisms within sequestra in $55 \%$ of patients with chronic osteomyelitis. ${ }^{4}$ It was found that these anaerobic organisms were more frequently isolated from the inside of the sequestra and were usually mixed isolates i.e two or more anaerobic isolates were isolated. The chronicity of the osteomyelitis was directly proportional to the frequency of anerobic isolates found.

In a retrospective study done on osteomyelitis by Lewis et al., it was found that 39 percent of patients with osteomyelitis had anaerobic infections. ${ }^{1}$ This serves to prove that anaerobes play a much larger role in osteomyelitis than known previously. It is likely that these infections are less reported due to poor awareness of their prevalence and the cumbersome methods of isolation.

However, these organisms were found as a part of the mixed flora which included gram positive cocci, gram negative bacilli and other anaerobes. This is consistent with the pattern of mixed infections reported in our case report. Our first patient was found to have anaerobic Prevotella infection from epidural abscess with associated Streptococcus bacteremia in a chronic tubercular vertebral osteomyelitis. Our second case was also found to have a combination of anaerobes comprising of Bacteroides, Peptoniphilus (Peptotreptococcus) and Clostridium with gram-negative Pseudomonas aeruginosa isolated from a psoas abscess secondary to a chronic tubercular vertebral osteomyelitis.

The predisposing factors contributing to anaerobic osteomyelitis usually include children, diabetes mellitus, oral infections or procedures, upper respiratory tract infections, trauma, peripheral neuropathy and complicated fractures. ${ }^{2}$ It is interesting to note that both our patients had no pre morbidities to predispose them to anaerobic osteomyelitis.

In a review by Raff and Melo of a large series of 193 anaerobic osteomyeltits cases collected from the world literature published between 1936 to 1976, it was the found that the most common anaerobe implicated in osteomyelitis was 

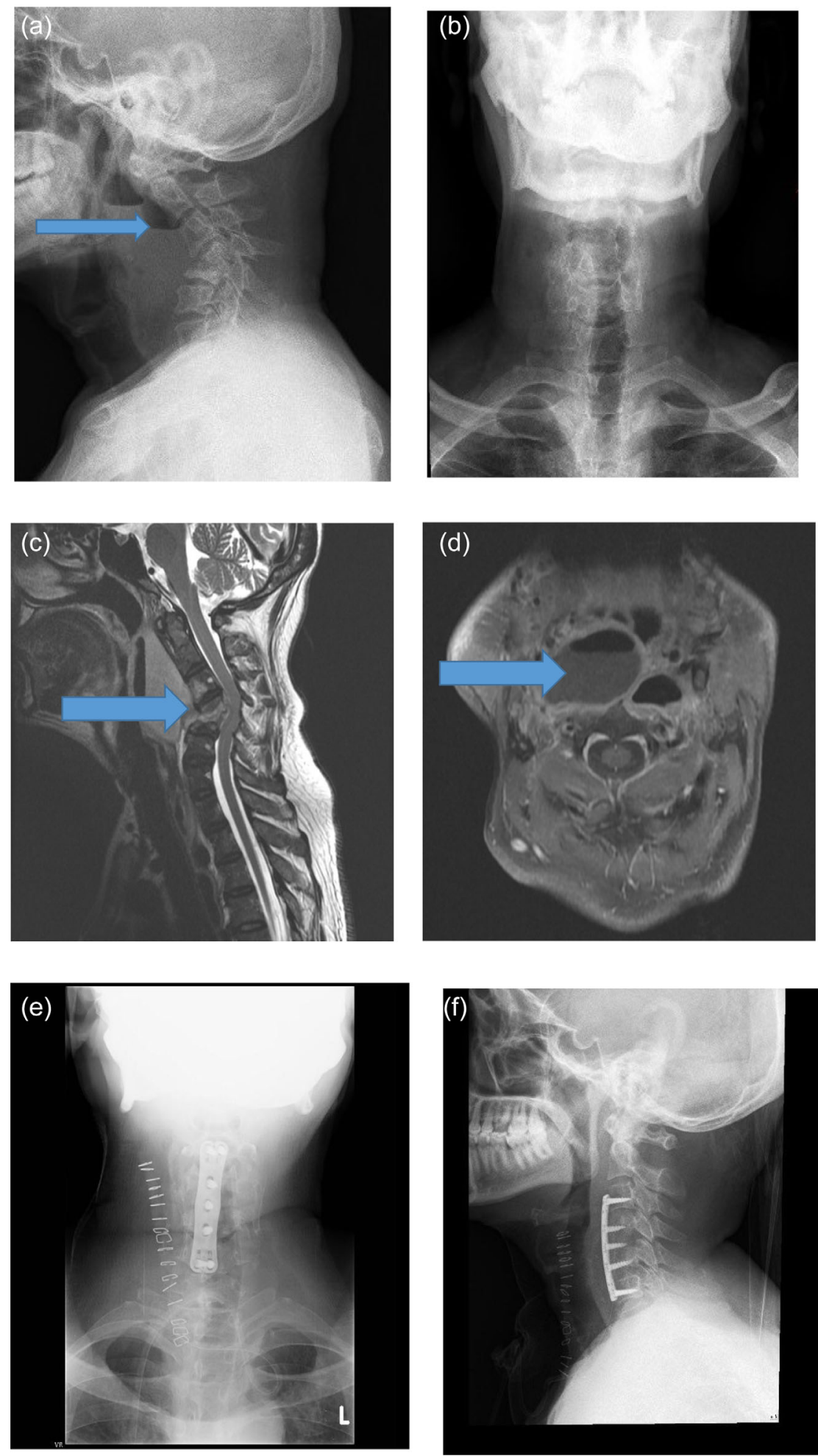

Figure 2. a: Radiograph cervical spine (lateral). b: Radiograph cervical spine (AP view). Arrow indicationg soft tissue shadow with air-fluid levels. c: MRI cervical spine (saggital view). d: MRI cervical spine (transverse view). Arrows indicating parapharyngeal collection with C4-5 spondylodiscitis. e: Cervical spine AP view. Postoperative radiographs. f: Cervial Spine lateral view. Post-operative radiographs. 
Table 1. Clinical characteristics of patients with Prevotella isolated from vertebral osteomyelitis (previous data).

\begin{tabular}{|c|c|c|c|c|c|}
\hline Authors & Age/sex & $\begin{array}{l}\text { Level of } \\
\text { osteomyelitis }\end{array}$ & $\begin{array}{l}\text { Organism } \\
\text { isolated }\end{array}$ & Risk factors & $\begin{array}{l}\text { Clinical } \\
\text { outcome }\end{array}$ \\
\hline Surbled et $\mathrm{al}^{8}$ & $44 / M$ & L5-S1 & P. melanogenica & none & Favourable \\
\hline Salavert et $\mathrm{al}^{9}$ & $27 / F$ & L2-L3 & P. melanogenica & IV drug use & Favourable \\
\hline Fukuoka et al ${ }^{10}$ & $60 / \mathrm{M}$ & T7-T8 & P. intermedia & Age & Favourable \\
\hline Schöber et $\mathrm{al}^{11}$ & $45 / F$ & L1-L2 & P. intermedia & None & Favourable \\
\hline Mukhyopadhyay et al ${ }^{12}$ & $35 / F$ & S1 & P. melanogenica & Dental cleaning & Favourable \\
\hline Salliot et $\mathrm{al}^{13}$ & $62 / \mathrm{M}$ & L5-S1 & Prevotella spp.* & Age, steroids & Favourable \\
\hline Purushothaman et $\mathrm{al}^{14}$ & $74 / F$ & L3-L4 & Prevotella spp.* & $\begin{array}{l}\text { Age, ovarian } \\
\text { malignancy }\end{array}$ & Favourable \\
\hline Huang et $\mathrm{al}^{15}$ & $73 / F$ & T12-L3 & P. melanogenica & Age, diabetes & $\begin{array}{l}\text { Patient } \\
\text { died }\end{array}$ \\
\hline Goyal et $\mathrm{al}^{16}$ & $68 / F$ & T6-T8 & P. oralis & $\begin{array}{l}\text { Age, } \\
\text { gastrointestinal } \\
\text { procedure }\end{array}$ & Favourable \\
\hline
\end{tabular}

*Species could not be identified.

Bacteroides followed by Peptostreptococcus and Fusobacterium. ${ }^{5}$ This trend was confirmed by a study by Ziment et al. who found the most common isolate being Bacteroides followed by Peptostreptococcus and Fusobacterium. ${ }^{6}$ This similar trend of occurrence was noted in a large study done on 134 cases of pyogenic osteomyelitis by Haider et al. between 1992 to $1993 .^{7}$ This trend agrees with our case of anaerobic osteomyelitis which showed a mixed anaerobic isolate of Bacteroides with Peptostreptococcus and Clostridium.

A review of literature on available data on anaerobic osteomyelitis with Prevotella isolates shows only nine cases reported till date. ${ }^{8-16}$ Among this only one case reports secondary Prevotella infection on chronic tubercular vertebral osteomyelitis. A summary of the data available on Anaerobic osteomyelitis with prevotella isolates are shown in Table 1.

Both our cases did not show any evidence of hematogenous osteomyelitis. This finding concurs with evidence found by in a review study on 61 cases of anaerobic osteomyelitis of long bones ${ }^{17}$ where $29.5 \%$ of the cases were hematogenous and $32.8 \%$ were exogenous osteomyelitis. The study by Lewis et al. also shows a preponderance towards exogenous osteomyelitis. ${ }^{1}$

Management of anaerobic osteomyelitis includes a two-pronged approach with adequate drainage of purulent material and parenteral administration of antibiotics for at least four to eight weeks. ${ }^{18}$ The antibiotics were decided according to the sensitivity pattern noted on culture.

Strengths and limitations: The strength of this case series is the similar clinical presentation of both the cases and the presence of aerobic and anaerobic infections in both of them. However, we were unable to follow up the second case beyond two months as he opted for care under a local orthopaedician due to travel constraints. This was one of the limitations of our case series. As a result of this adherence and tolerance of the treatment could not be assessed in the second case.

\section{Conclusion}

Though tubercular vertebral osteomyelitis is usually a diagnosis in itself, it should not hinder us from considering secondary infections (both aerobic and anaerobic) complicating the osteomyelitis. Further, the presence of air-fluid levels on imaging studies and the presence of foul smell during operative exploration of the spine must arouse the suspicion of an anaerobic co-infection. Isolation and treatment of these organisms are crucial as they may hamper the clinical outcome of the primary TVO. Therefore, the cocktail of organisms, both aerobic and anaerobic isolated in these cases highlights the importance of suspecting, isolating and treating these lurking organisms in cases of tuberculosis of spine. 


\section{Patient perspective}

1. I was suffering from severe back ache for over 2 years with recurrent fever. I was unable to continue my studies also as sitting in class was very painful. I received treatment at several local hospitals but the fever persisted and the pain was not alleviated. Following admission at Kasturba hospital, Manipal, I underwent an MRI of my spine and the doctors found the source of infection in my spine. I underwent a decompression surgery with treatment with TB medicines and intravenous medications at the hospital. The fever subsided in the first week following treatment. Gradually I was able to move around with support and at my 3 month follow-up, I walked without pain to the hospital. I am much better now and have rejoined college to continue my studies.

2. I had neck stiffness and pain for many years. The pain radiated to my arms and I struggled to sit and work for long hours with my neck bent forward. In the hospital, a team of doctors examined me and suspected a spine infection. An MRI confirmed infection of the neck bones and pus collections. I underwent a surgery on my neck and received a long course of medicines in the hospital and at home. I noted a significant improvement in my pain and fever. I was symptom free by my 2-month follow-up at the hospital.

1. Lewis RP, Sutter VL, Finegold SM: Bone infections involving anaerobic bacteria. Medicine. 1978; 57(4): 279-306. PubMed Abstract | Publisher Full Text

2. Brook I, Frazier EH: Anaerobic osteomyelitis and arthritis in a military hospital: A 10 year experience. Am. J. Med. 1993; 94(1): 21-28.

PubMed Abstract | Publisher Full Text

3. Bick E: The source book of orthopaedics. Baltimore: The Williams \& wilkins co; 1948.

4. Taylor K, Davies M: Persistence of bacteria within sequestra. Ann Surg. 1917; 66: 522-528.

PubMed Abstract | Publisher Full Text | Free Full Text

5. Raff MJ, Melo JC: Anaerobic osteomyelitis. Medicine. 1978; 57: 83-103.

Publisher Full Text

6. Ziment I, Miller LG, Finegold SM: Nonsporulating anaerobic bacteria in osteomyelitis. Antimicrob. Agents Chemother. 1967; 7: 77-85.

PubMed Abstract

7. Haider AM, Sundus SB, Thamer AH: Anaerobic osteomyelitis. East Mediterr. Health J. 1996; 2: 494-500.

Publisher Full Text

8. Surbled M, Perrier-Creach C, Rabouille Y, et al.: Spondylodiscitis caused by Bacteroides melaninogenicus. Presse Med. 1992; 21: 1870-1871.

9. Salavert M, Navarro V, Roig P, et al.: Vertebral osteomyelitis by Prevotella melaninogenica, Candida albicans, and Mycobacterium tuberculosis in a intravenous drug addict. Enferm. Infecc. Microbiol. Clin. 1997; 15: 117-119.

10. Fukuoka M, Aita K, Aoki Y, et al.: Pyogenic vertebral osteomyelitis caused by Prevotella intermedia. J. Infect. Chemother. 2002; 8 : 182-184.

PubMed Abstract | Publisher Full Text
11. Schöber W, Horger M, Niehues D: One case of Gram-negative anaerobic spondylodiscitis with Prevotella intermedia. Arch Orthop. Trauma Surg. 2003; 123: 436-438. PubMed Abstract | Publisher Full Text

12. Mukhopadhyay S, Rose F, Frechette V: Vertebral osteomyelitis caused by Prevotella (Bacteroides) melaninogenicus. South. Med. J. 2005; 98: 226-228. PubMed Abstract | Publisher Full Text

13. Salliot C, Lavie F, Azria A, et al.: Retroperitoneal fibrosis secondary to spondylodiscitis after infection with Prevotella. J. Rheumatol. 2005; 32: 957-958. PubMed Abstract

14. Purushothaman B, Lakshmanan P, Gatehouse S, et al.: Spondylodiscitis due to Prevotella associated with ovarian mass - a rare case report and review of literature. World Neurosurg. 2010; 73: 119-122. PubMed Abstract | Publisher Full Text

15. Huang CR, Lu CH, Chuang YC, et al.: Clinical characteristics and therapeutic outcome of Gram-negative bacterial spinal epidural abscess in adults. J. Clin. Neurosci. 2011; 18: 213-217. Publisher Full Text

16. Hemant G, Shitij A, Sneha M, et al.: Vertebral osteomyelitis and epidural abscesses caused by Prevotella oralis: a case report. Braz. J. Infect. Dis. 2012 Dec; 16(6): 594-596. Publisher Full Text

17. Templeton WC, et al.: Anaerobic osteomyelitis of long bones. Rev. Infect. Dis. 1983; 5(4): 692-712. PubMed Abstract | Publisher Full Text

18. Brook I: Microbiology and management of joint and bone infections due to anaerobic bacteria. Journal of Orthopaedic Science: Official Journal of the Japanese Orthopaedic Association. 2008; 13(2): 160-169.

PubMed Abstract | Publisher Full Text 


\section{Open Peer Review}

\section{Current Peer Review Status: $\mathrm{X}$ ?}

\section{Version 1}

Reviewer Report 22 March 2022

https://doi.org/10.5256/f1000research.78143.r123606

(C) 2022 Rahmathulla G. This is an open access peer review report distributed under the terms of the Creative Commons Attribution License, which permits unrestricted use, distribution, and reproduction in any medium, provided the original work is properly cited.

\section{Gazanfar Rahmathulla}

Department of Neurosurgery, University of Florida Health, University of Florida Health Jacksonville, FL, USA

The authors present a case report on 2 cases of vetebral osteomyelitis of the lumbar and cervical spine where the patients have co-existing mycobacterial and aerobic/anerobic infections. Both cases had surgical intervention with short term reported good outcomes.

Pros - interesting article discussing the presence of overlapping bacterial infections in vertebral osteomyelitis.

Cons - short follow up, no discussion about the length of anti-TB medications used, absence of radiological follow-up.

The authors can write their article in standard format of introduction, material / methods where they can note the consent requirement rather than before the discussion.

The authors should expand their discussion to explain the duration of anti-TB treatment in the presence of treatment for other organisms, their follow-up strategy and explain how the patient could have got these poly organisms to infect the spine.

In the MRI images for case 1, the descriptions can be more clear and detailed, as they only show the psoas abscess but would better serve the readers by focusing on the vertebral osteomyelitis components as well.

The authors should place another table with literature discussing TB with other infections and all the papers available on this topic. There will not be many and hence this paper would then be strengthened.

Grammatical changes required in different paragraphs.

Is the background of the cases' history and progression described in sufficient detail? 
Yes

Are enough details provided of any physical examination and diagnostic tests, treatment given and outcomes?

No

Is sufficient discussion included of the importance of the findings and their relevance to future understanding of disease processes, diagnosis or treatment?

Yes

Is the conclusion balanced and justified on the basis of the findings?

Yes

Competing Interests: No competing interests were disclosed.

Reviewer Expertise: Traumatic brain and spine injury, spinal surgery and associated pathology, brain tumors, radiosurgery

I confirm that I have read this submission and believe that I have an appropriate level of expertise to confirm that it is of an acceptable scientific standard, however I have significant reservations, as outlined above.

\section{Author Response 26 Oct 2022}

Cynthia Sukumar, Manipal University, Manipal, India

The case report has been revised to include physical examination, diagnostic tests, details of treatment and clinical outcome. Additional radiological images have been included for case 1 to indicate spondylodiscitis.

Literature search to find similar cases with tubercular vertebral osteomyelitis and anaerobic infection was also done and details included in the manuscript.

Competing Interests: I have no competing interests.

Author Response 26 Oct 2022

Cynthia Sukumar, Manipal University, Manipal, India

\section{APPROVED WITH RESERVATIONS}

The authors can write their article in standard format of introduction, material / methods where they can note the consent requirement rather than before the discussion.- revised

The authors should expand their discussion to explain the duration of anti-TB treatment in the presence of treatment for other organisms, their follow-up strategy and explain how the patient could have got these poly organisms to infect the spine.- Revised 
In the MRI images for case 1, the descriptions can be more clear and detailed, as they only show the psoas abscess but would better serve the readers by focusing on the vertebral osteomyelitis components as well. - images included

The authors should place another table with literature discussing TB with other infections and all the papers available on this topic. There will not be many and hence this paper would then be strengthened.

Grammatical changes required in different paragraphs.

Is the background of the cases' history and progression described in sufficient detail? Yes

Are enough details provided of any physical examination and diagnostic tests, treatment given and outcomes?

\section{No... Details provided in the revised manuscript}

Is sufficient discussion included of the importance of the findings and their relevance to future understanding of disease processes, diagnosis or treatment? Yes

Is the conclusion balanced and justified on the basis of the findings? Yes

Competing Interests: No competing interests.

Reviewer Report 10 March 2022

https://doi.org/10.5256/f1000research.78143.r121806

(c) 2022 Maatallah K. This is an open access peer review report distributed under the terms of the Creative Commons Attribution License, which permits unrestricted use, distribution, and reproduction in any medium, provided the original work is properly cited.

\section{Kaouther Maatallah}

Rheumatology Department, Med Kassab institute of orthopedics, Tunisia Faculty of medicine of Tunis, University Tunis el Manar, Manouba, Tunisia

These are two cases of tuberculosis of the spine complicated by aerobic and anaerobic infections. The first is lumbar spondylodiscitis in a young woman. A second case is a middle-aged man with C4-5 cervical spondylodiscitis. Both cases improved after receiving antibiotic treatment and decompression surgery.

In both cases, the chest radiography findings were not detailed. In the first case, no MRI images 
showed spondylodiscitis.

The reason for the surgical treatment is not justifiable since, in both cases, there was no motor deficit.

Is the background of the cases' history and progression described in sufficient detail? Yes

Are enough details provided of any physical examination and diagnostic tests, treatment given and outcomes?

Partly

Is sufficient discussion included of the importance of the findings and their relevance to future understanding of disease processes, diagnosis or treatment?

Partly

Is the conclusion balanced and justified on the basis of the findings?

Partly

Competing Interests: No competing interests were disclosed.

Reviewer Expertise: Rheumatology

I confirm that I have read this submission and believe that I have an appropriate level of expertise to state that I do not consider it to be of an acceptable scientific standard, for reasons outlined above.

Author Response 26 Oct 2022

Cynthia Sukumar, Manipal University, Manipal, India

NOT APPROVED

These are two cases of tuberculosis of the spine complicated by aerobic and anaerobic infections. The first is lumbar spondylodiscitis in a young woman. A second case is a middleaged man with C4-5 cervical spondylodiscitis. Both cases improved after receiving antibiotic treatment and decompression surgery.

In both cases, the chest radiography findings were not detailed. In the first case, no MRI images showed spondylodiscitis.- MRI images included

The reason for the surgical treatment is not justifiable since, in both cases, there was no motor deficit.

Is the background of the cases' history and progression described in sufficient detail? Yes

Are enough details provided of any physical examination and diagnostic tests, 
treatment given and outcomes?

Partly - Details included

Is sufficient discussion included of the importance of the findings and their relevance to future understanding of disease processes, diagnosis or treatment?

Partly - Discussion has been revised as per your suggestions

Is the conclusion balanced and justified on the basis of the findings?

Partly - Revised

Competing Interests: No competing interests

The benefits of publishing with F1000Research:

- Your article is published within days, with no editorial bias

- You can publish traditional articles, null/negative results, case reports, data notes and more

- The peer review process is transparent and collaborative

- Your article is indexed in PubMed after passing peer review

- Dedicated customer support at every stage

For pre-submission enquiries, contact research@f1000.com 